\title{
Gastrointestinal Parasitosis: Histopathological Insights to Rare But Intriguing Lesions of the Gastrointestinal Tract
}

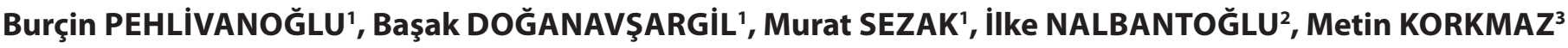 \\ 'Department of Pathology, Ege University Faculty of Medicine, IZMIR, TURKEY \\ ${ }^{2}$ Department of Pathology and Immunology, Washington University School of Medicine, St. Louis, MISSOURI, USA \\ ${ }^{3}$ Department of Parasitology, Ege University Faculty of Medicine, IZMIR, TURKEY
}

\begin{abstract}
Objective: Gastrointestinal parasitosis is a significant cause of morbidity and mortality. Definitive diagnosis is usually made by stool tests and/or serology but may require tissue evaluation. Although pathologists are usually familiar with common parasites, it is not well established whether the diagnosis could be suspected without seeing the "parasite" itself.

Material and Method: Resection or biopsy specimens of 32 cases with Giardia intestinalis ( $\mathrm{n}=20)$, Enterobius vermicularis ( $\mathrm{n}=5)$, Entamoeba histolytica $(n=4)$, Fasciola hepatica $(n=1)$, Strongyloides spp. $(n=1)$ and Taenia saginata $(n=1)$ infections were retrospectively re-evaluated for accompanying mucosal changes, and compared with nonparametric tests.

Results: The most common changes were congestion (65.6\%) and eosinophilic infiltration (50\%). Chronic active mucosal inflammation accompanied $37.5 \%$ of the cases. More than 10 eosinophils/HPF were present in $43.8 \%$. Only one case of G. intestinalis, E. vermicularis, E. histolytica, and F. hepatica showed more than 50 eosinophils/HPF. Mucosal architectural abnormalities were present in 34.4\%. Granulomas, giant cells and Charcot-Leyden crystals were only seen accompanying F. hepatica. No statistically significant difference was found between parasite subspecies regarding presence of inflammation, lymphoid aggregates, architectural distortion, congestion, ulceration and increase of eosinophils

Conclusion: Parasites induce nonspecific inflammation, slight mucosal architectural changes, mild eosinophilic infiltrate or granuloma formation. They may cause ulceration, bowel obstruction or perforation. Parasitosis should also be considered when evaluating cases mimicking inflammatory bowel disease, celiac disease or those that do not fulfill diagnostic criteria.
\end{abstract}

Key Words: Histopathology, Parasitic diseases, Eosinophilia, Infection, Gastrointestinal tract

\section{INTRODUCTION}

Parasitic infections of the gastrointestinal (GI) tract are significant causes of morbidity and mortality. Although they are particularly more common in underdeveloped regions with poor sanitary conditions, they are prevalent throughout the whole world. Clinical presentation may vary depending on the parasite type and the affected parts of the GI tract. Parasites can survive in the GI tract for years without causing any symptoms. However, occasionally they may cause serious clinical presentations. Although a diagnostic approach can be made on clinical grounds, they may present important diagnostic challenges as they can mimic important GI pathologies such as eosinophilic gastroenteritis (1), gluten sensitive enteropathy (2) or inflammatory bowel diseases (3). The definitive diagnosis is usually made by stool cultures, stool microscopy and/ or serology. However, some cases may require tissue evaluation in order to rule out other GI tract pathologies.

(Turk Patoloji Derg 2016, 32:82-90)

Received : 20.11.2015 Accepted : 28.12.2015
Therefore, the pathologist should also be aware of the features of parasitic infections.

In this context, pathologists are usually familiar with common types of protozoa or at least can identify the microorganism as "a parasite" and may refer to a parasitologist for a more proper identification. On the other hand, microbiologists/parasitologists generally do not have adequate knowledge about direct microscopic visualization of pathogens in biopsy samples (4).

Currently, most of the large series on human GI parasites usually focus on stool microscopy and there is very limited literature background dedicated to histological features alone. Besides it is not well known whether there are specific clues to suspect a parasitic infection or not, especially in cases where the microorganism is either very sparse or not visualized on small biopsy material or when there is no relevant clinical information upon the possibility of a parasitic infection.

Correspondence: Burçin PEHLIVANOĞLU

Adıyaman Üniversitesi Eğitim ve Araştırma Hastanesi, Patoloji Bölümü 02000 ADIYAMAN, TURKEY

E-mail: burcinp@yahoo.com Phone: +90 4162161015 
In this study, we aimed to evaluate the accompanying histological features seen in tissue samples and to determine the effectiveness of these features for providing information to suspect a parasitic infection.

\section{MATERIALS and METHODS}

Biopsy or resection specimens of 32 consecutive patients diagnosed between 2000 and 2011 were included in the study. The study group was composed of cases with Giardia intestinalis $(\mathrm{n}=20)$, Enterobius vermicularis $(\mathrm{n}=5)$, Entamoeba histolytica $(\mathrm{n}=4)$, Fasciola hepatica $(n=1)$, Strongyloides spp. $(\mathrm{n}=1)$ and Taenia saginata $(n=1)$ infections. Cases with E. vermicularis, T. saginata and F.hepatica were evaluated with their resection specimens while the rest of the cases were evaluated with their endoscopic biopsy materials retrospectively in respect to accompanying mucosal changes consisting of the presence of chronic and acute inflammation, increase of eosinophils, congestion, mucosal ulceration, necrosis, perforation, lymphoid aggregates, cryptic architectural distortion, granuloma formation, calcification, hyalinization, and presence of giant cells and/or Charcot-Leyden crystals. Eosinophil density was determined as the number of eosinophils in one high power field (HPF, $\mathrm{x} 40$ objective) and later on dichotomized into two groups as cases presenting over 10 or 50 eosinophils per one HPF for statistical purposes. Statistical analysis was performed using a computer based program, SPSS version 13.0. Observed changes were correlated with the parasite type by nonparametric tests (chi-square and Fisher's exact test when appropriate). The protozoon categories with only one representative case were excluded from statistical analysis.
However, the observed changes were provided in the results and discussed accordingly.

\section{RESULTS}

The clinical features of the cases are summarized in Table I and accompanying histological changes according to the protozoon type are given in Table II.

Fifty-three percent of the cases were female and median age was $38 \pm 19.3$ years (range $4-61$ years). The median age was lower in females compared to males $(28.7 \pm 17.5$ versus $42.9 \pm 19.2$, respectively), and in cases with $E$. vermicularis $(15 \pm 13.8)$ although statistically insignificant. Overall $12.5 \%$ of the cases had accompanying malignancies such as lymphoma, stomach and colon cancers and $22 \%$ had a chronic disease such as common variable immunodeficiency (CVID), chronic Helicobacter pylori (HP) gastritis or Familial Mediterranean fever (FMF).

The taeniasis case (65-year-old male) presented to the hospital with small bowel perforation due to an obstructing mass suspicious for malignancy (Figure 1). There was no known previous history of parasitosis in that case as well as another case with perforated acute appendicitis with $E$. vermicularis infection (15 year-old female). The case with F. hepatica (46-year-old male) was also complicated with colon perforation.

Apart from the remaining four appendectomies, the rest of the cases were diagnosed with their biopsy materials by identification of the parasite itself. Retrospective evaluation revealed that the most commonly observed mucosal change was congestion (65.6\%) that was accompanied by acute mucosal inflammation in $37.5 \%$ of the cases.

Table I: Clinical features of the cases

\begin{tabular}{|c|c|c|c|c|c|c|}
\hline Type of parasite & $\mathbf{n}$ & $\%$ & Age (Median \pm SD, Range) & Gender (M/F) & Localisation (n) & $\begin{array}{c}\text { Accompanying disease } \\
\text { ( } \mathrm{n} \text { of cases) }\end{array}$ \\
\hline Giardia lamblia & 20 & 62.5 & $35.5 \pm 19.4(4-65)$ & $7 \mathrm{M}, 13 \mathrm{~F}$ & Duodenum (20) & $\begin{array}{l}\text { CVID (4), HP gastritis } \\
\text { (6), CRF (1), colon cancer } \\
\text { (1), stomach cancer (1), } \\
\text { lymphoma (1), none (6) }\end{array}$ \\
\hline $\begin{array}{l}\text { Enterobius } \\
\text { vermicularis }\end{array}$ & 5 & 15.6 & $15 \pm 13.8(5-41)$ & $1 \mathrm{M}, 4 \mathrm{~F}$ & Appendix (5) & FMF (1), none (4) \\
\hline $\begin{array}{l}\text { Entamoeba } \\
\text { histolytica }\end{array}$ & 4 & 12.5 & $49 \pm 14.8(25-60)$ & $4 \mathrm{M}$ & $\begin{array}{l}\text { Colon (2), } \\
\text { Rectum (2) }\end{array}$ & None \\
\hline Strongyloides & 1 & 3.1 & 59 & $1 \mathrm{M}$ & Stomach (1) & Marginal zone lymphoma \\
\hline Taenia saginata & 1 & 3.1 & 65 & $1 \mathrm{M}$ & Small bowel (1) & None \\
\hline Fasciola hepatica & 1 & 3.1 & 46 & $1 \mathrm{M}$ & Colon (1) & Cholecystitis \\
\hline
\end{tabular}

CVID: Common variable immune deficiency, HP: Helicobacter Pylori, CRF: Chronic renal failure, FMF: Familial Mediterranean fever. 
Table II: Distribution of histopathological features of the cases according to protozoon type

\begin{tabular}{|c|c|c|c|c|c|c|c|c|c|c|c|c|}
\hline Histopathological Change & 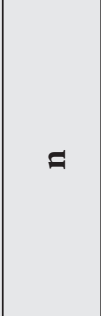 & de & 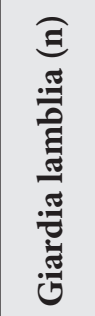 & $a^{0}$ & 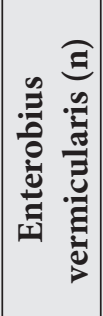 & de & 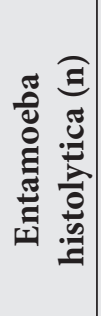 & de & 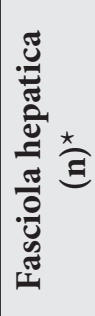 & 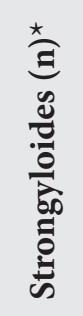 & 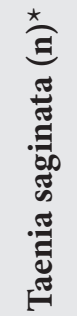 & a \\
\hline & $\begin{array}{c}32 \\
\text { cases } \\
\end{array}$ & 100 & $\begin{array}{c}20 \\
\text { cases } \\
\end{array}$ & & $\begin{array}{c}5 \\
\text { cases } \\
\end{array}$ & & $\begin{array}{c}4 \\
\text { cases } \\
\end{array}$ & & $\begin{array}{c}1 \\
\text { case } \\
\end{array}$ & $\begin{array}{c}1 \\
\text { case } \\
\end{array}$ & $\begin{array}{c}1 \\
\text { case }\end{array}$ & \\
\hline Congestion/hemorrhage & 21 & 65.6 & 12 & & 3 & & 4 & & 1 & 0 & 1 & .369 \\
\hline Eosinophilic infiltration $>10 / 1 \mathrm{HPF}$ & 16 & 50.0 & 12 & 60 & 0 & 0 & 2 & 50 & 0 & 1 & 1 & .134 \\
\hline Lymphoid aggregates & 15 & 46.9 & 8 & 40 & 3 & 60 & 2 & 50 & 1 & 0 & 1 & .565 \\
\hline Chronic active inflammation & 12 & 37.5 & 4 & 20 & 3 & 60 & 3 & 75 & 1 & 0 & 1 & .74 \\
\hline $\begin{array}{l}\text { Mucosal architecture changes (i.e. villous } \\
\text { blunting, crypt distortion) }\end{array}$ & 11 & 34.4 & 7 & 35 & 1 & 20 & 2 & 50 & 0 & 0 & 1 & .571 \\
\hline Chronic lymphoplasmacytic infiltration & 9 & 28.3 & 0 & 0 & 5 & 100 & 2 & 50 & 1 & 0 & 1 & .280 \\
\hline Erosion/ulceration & 6 & 18.8 & 2 & 10 & 2 & 40 & 2 & 50 & 0 & 0 & 0 & .332 \\
\hline Eosinophilic infiltration $>50 / 1 \mathrm{HPF}$ & 4 & 12.5 & 1 & 5 & 1 & 20 & 1 & 25 & 1 & 0 & 0 & .103 \\
\hline Hyalinization & 4 & 12.5 & 0 & 0 & 3 & 60 & 0 & 0 & 0 & 0 & 1 & .001 \\
\hline Necrosis & 3 & 9.4 & 0 & 0 & 1 & 20 & 0 & 0 & 1 & 0 & 1 & .000 \\
\hline Perforation & 3 & 9.4 & 0 & 0 & 1 & 20 & 0 & 0 & 1 & 0 & 1 & .000 \\
\hline Metaplasia (Gastric/intestinal) & 2 & 6.3 & 2 & 10 & 0 & 0 & 0 & 0 & 0 & 0 & 0 & .937 \\
\hline Abscess formation & 2 & 6.3 & 0 & 0 & 1 & 20 & 0 & 0 & 1 & 0 & 0 & ** \\
\hline Calcification & 2 & 6.3 & 0 & 0 & 2 & 40 & 0 & 0 & 0 & 0 & 0 & .042 \\
\hline Granuloma formation & 1 & 3.1 & 0 & 0 & 0 & 0 & 0 & 0 & 1 & 0 & 0 & $* *$ \\
\hline Giant cells & 1 & 3.1 & 0 & 0 & 0 & 0 & 0 & 0 & 1 & 0 & 0 & $* *$ \\
\hline Charcot-Leyden crystals & 1 & 3.1 & 0 & 0 & 0 & 0 & 0 & 0 & 1 & 0 & 0 & $* *$ \\
\hline
\end{tabular}

* The protozoon categories with only one representative case were included in the Table to give an idea about the spectrum of histological changes.

** The histological features observed solely in those cases were excluded from statistical analysis.

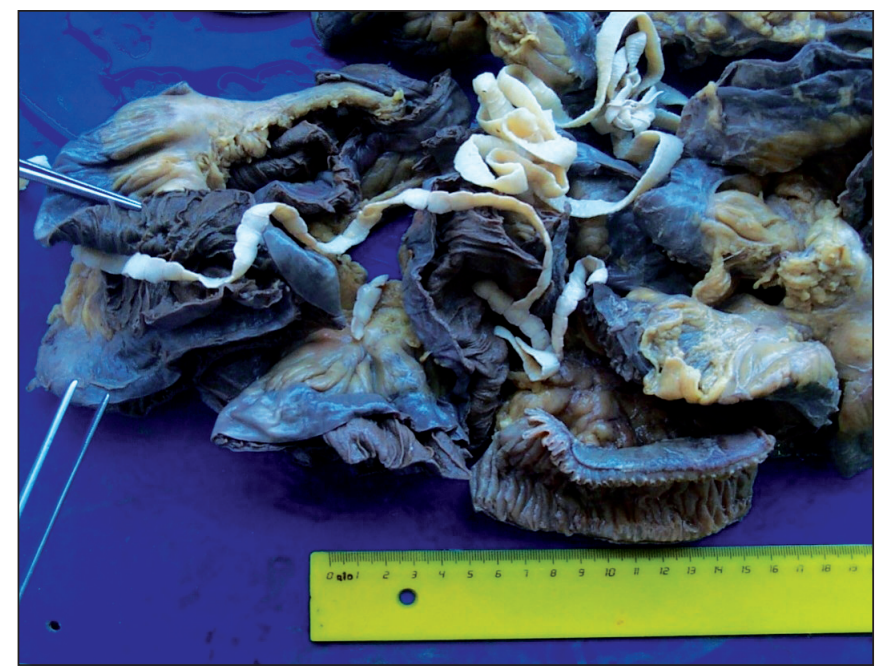

Figure 1: Gross image of ischemic colon containing a pile of Taenia saginata proglottid.
Mucosal erosion and ulceration (18.8\%) was a feature of amebiasis (Figure 2A,B), enterobiasis and giardiasis, possibly due to complicating acute appendicitis for enterobiasis (Figure 3A-D) and gastric mucinous metaplasia in duodenum for giardiasis. Non-Peyer patch lymphoid aggregates (46.9\%) (Figure 4A,B) and eosinophilic infiltration (50\%) were the other common findings. The gastric mucosa with Strongyloides was almost normal other than the presence of the parasite itself (Figure 5A-D). The number of eosinophils differed between 2 and 75/HPF (median 22.31 \pm 22.4 ). More than 10 and 50 eosinophils were present in $43.8 \%$ and $12.5 \%$ of the cases, respectively. Although statistically insignificant, eosinophilic infiltration was a prominent feature observed in $85 \%(\mathrm{n}=13)$ of giardiasis cases as well as faint villous blunting observed in 7 (35\%) of these cases (Table II). None of them exhibited increased intraepithelial lymphocytosis 


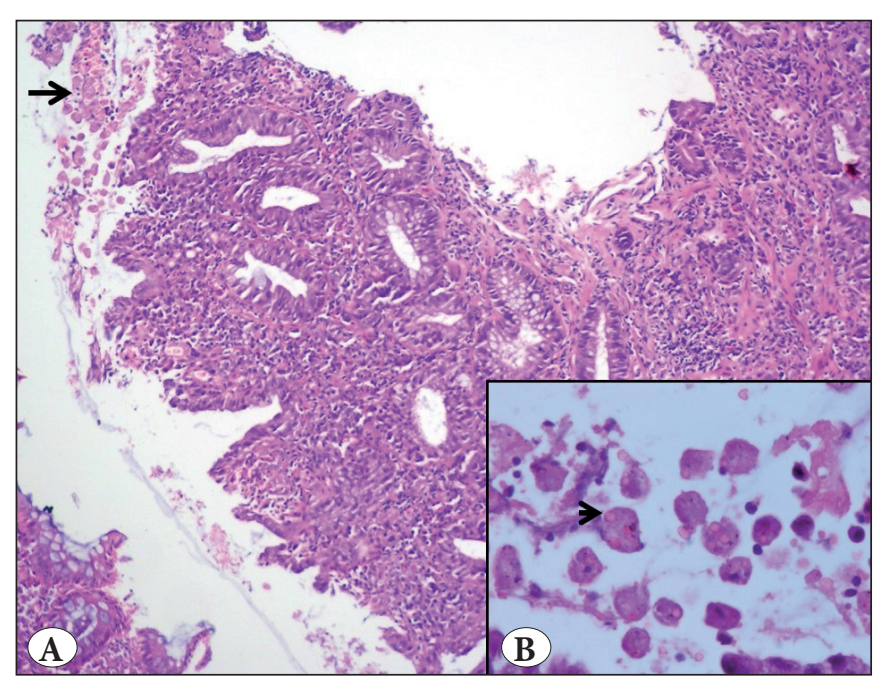

Figure 2: A) Entamoeba spp. in a colonoscopic biopsy (long arrow). Note the erosion, mixed inflammatory infiltration and mucosal architectural irregularity mimicking a chronic inflammatory bowel disease (H\&E; x100). B) "Engulfed" erythrocytes within trophozoites are seen (short arrow) (H\&E; x400).

raising suspicion for gluten sensitive enteropathy (Celiac disease). G. intestinalis trophozoites were seen between adjacent villi (Figure 4 C,D). Granulomas, giant cells and Charcot-Leyden crystals were only seen accompanying F. hepatica (Figure 6A-D). Hyalinization was especially prominent in enterobiasis $(\mathrm{p}=0.001)$ and in sole case of taeniasis. Two cases with $E$. vermicularis showed calcification ( $\mathrm{p}=0.042)$. However, no significant difference was found between parasite subspecies regarding presence of chronic and acute inflammation, lymphoid aggregates, architectural distortion, congestion, ulceration and increase of eosinophils.

\section{DISCUSSION}

The correct and timely diagnosis of infectious disease is essential for immediate onset of the effective treatment. Stool microscopy is a faster method but is not relevant in all types of parasites and stool cultures and serological tests may be useful in supporting diagnosis $(5,6)$. In this respect, histopathological approach is also informative in identifying the pathogen but as an invasive method it is unnecessary in most cases. Thus, biopsies are usually done for diagnostic purposes and for excluding other entities while resections are only used in complicated cases.

However, there are incidents where the histopathological diagnosis precedes the clinical diagnosis, as seen in some cases of our series. Among the cases presented herein, there was no prior diagnosis in taeniasis and one of the cases of enterobiasis until their admission with small bowel and appendix perforation. Strongyloides spp. in the stomach was also an incidental finding in endoscopic biopsy performed to rule out recurrent lymphoma. Similarly, G. intestinalis was a concomitant but clinically unsuspected finding diagnosed while the cases were being investigated for other diseases such as gastric adenocarcinoma, colonic mucinous adenocarcinoma, lymphoma, FMF and CVID (in four cases).

The tissue diagnosis is usually straightforward when the causative agent can be easily identified in slides or even visible in macroscopic materials as seen in taeniasis. The challenging issue is the identification of the parasite when there is a very limited number of the trophozoites, cyst, ova, and larval or adult forms on the slide. Although the role of the pathologist is usually to confirm the clinical suspicion in one way or the other, it may even not be possible to make a differential diagnosis between an infectious pathology and chronic GI tract disorders.

The initial step for the diagnosis of bacterial, fungal and viral infections in tissue sections is the identification of inflammation and determination of the dominant cell type (4). Polymorphonuclear leukocyte (PNL) infiltration is usually more prominent in bacterial and fungal infections while viral infections usually cause a lymphocytic reaction. However, although the efficacy of histopathological examination in the diagnosis of infectious disease has been well established, there is no specific tissue response or dominant inflammation type described for parasitic diseases.

G. intestinalis was the most common pathogen (62.5\%) in this study followed by E. vermicularis (15.6\%) and E. histolytica (12.5\%). Although this cannot be interpreted as reliable epidemiological data due to limited number of cases examined, it makes sense as those three parasites also appear to be the most common cause of GI parasitosis while the rank of prevalence may show geographical variations (6) and may even vary among different ethnic or age groups living in the same area (5).

In this study, an overall "normal" appearing mucosa was only observed in G. intestinalis infections. In almost $40 \%$ of the cases only lymphoid aggregates, and slight villous blunting which can be attributable to presence of lymphoid aggregates were observed. Previous reports on the effect of G. intestinalis on villous architecture are controversial (2, 7). It causes brush border abnormalities, but recent studies indicate that the villous architecture is usually preserved if the concomitant diseases are disregarded (7). Although giardiasis is an important diagnostic consideration in 

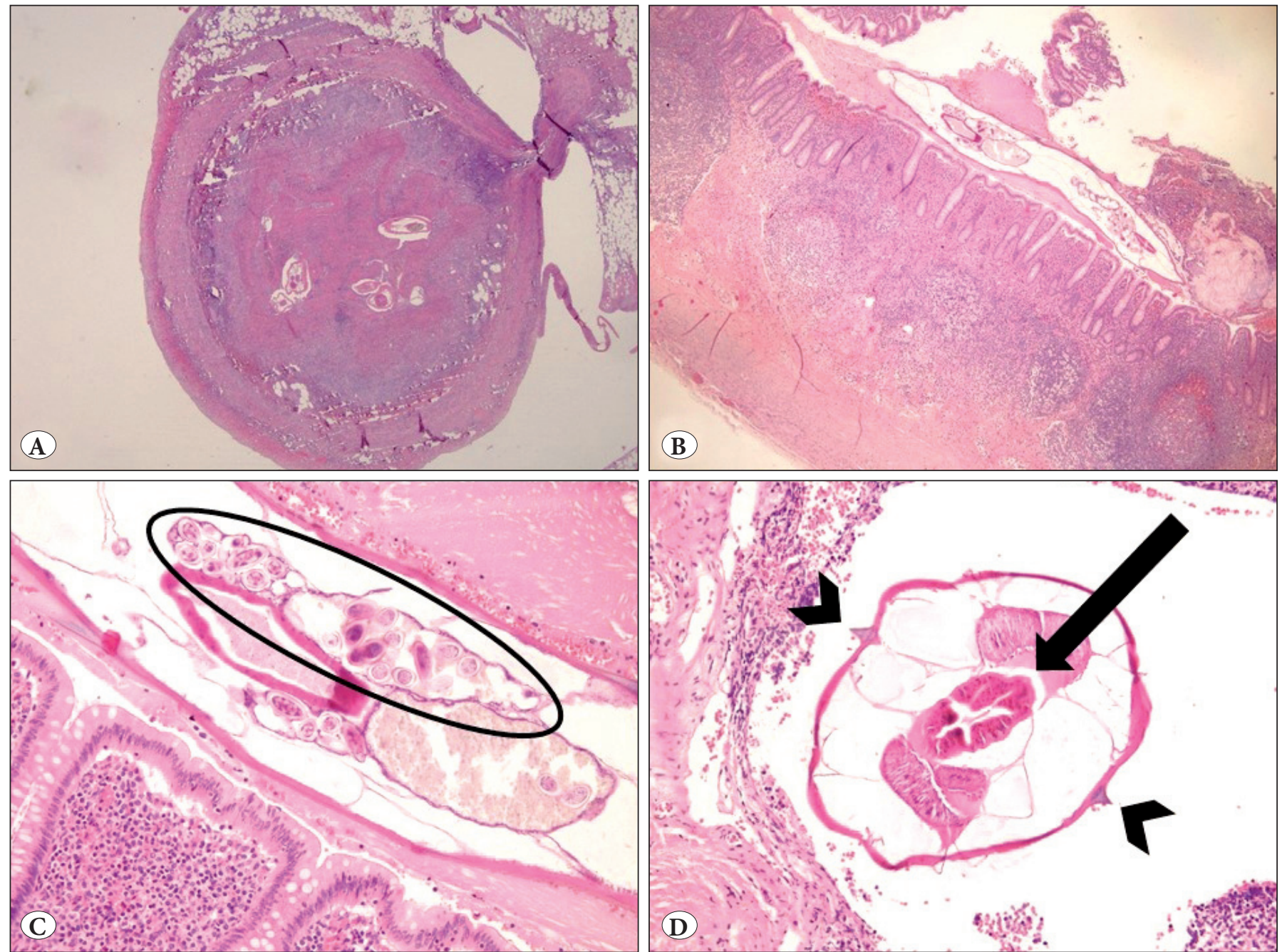

Figure 3: Enterobius vermicularis causing acute appendicitis. E. vermicularis in lumen, A) Cross section image (H\&E; x20). B) Longitudinal section image (H\&E; x40). C) Eggs, circled in yellow (H\&E; x200). D) E. vermicularis' alae (arrow heads) and esophagus (long arrow) (H\&E; x200).

cases with suspected gluten sensitive enteropathy, villous atrophy, intraepithelial lymphocytosis and/or crypt hyperplasia are also extremely rare (7) and when present should not be attributed to giardiasis unless all other diseases are eliminated. We did not observe intraepithelial lymphocytosis or crypt hyperplasia either. Mild duodenitis with predominant eosinophilic infiltration was previously defined but is less prevalent (8). This is consistent with our findings as we also observed active inflammation in $20 \%$ of the cases. However, it was probably due to peptic duodenitis as it was accompanied by gastric mucinous metaplasia and erosions, so it is not easy to consider presence of epithelial infiltrating PNL as a tissue response to giardiasis. In this context, eosinophilic infiltration is noteworthy as it was prominent in $60 \%$ of the cases even though there was more than 50/HPF in only one case. Clinical history of diarrhea or CVID, with lack or paucity of plasma cells in lamina propria, are also important clues for more careful scanning for possible Giardia colonization. The presence of CVID in $20 \%$ of giardiasis cases in our series is also noteworthy but not surprising considering the increased tendency for giardiasis in CVID patients (9). However, this finding should be interpreted with caution since CVID is a rare disease and this study was conducted at a tertiary referral center. While the uncomplicated cases are usually diagnosed and treated in primary care units, cases that may require further analysis or require endoscopic biopsies are referred for consultation.

Notably, there were only few G. intestinalis trophozoites in a small area between two adjacent villi in one of the cases that were sent for a second opinion to rule out Celiac disease. Therefore, even though G. intestinalis has very typical pear- 

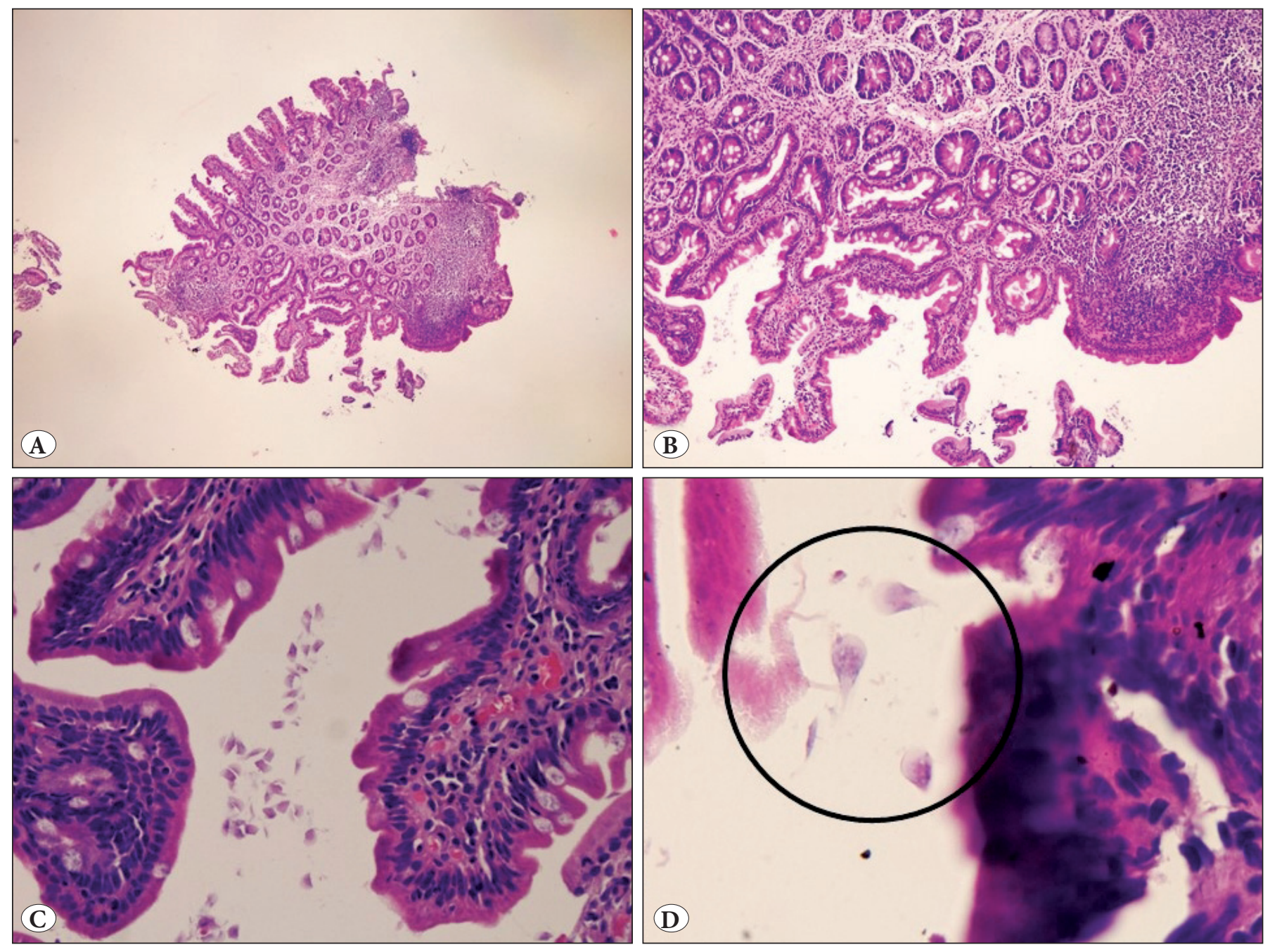

Figure 4: A-B) Giardia intestinalis trophozoites in a duodenal biopsy. Note the lymphoid follicle and mild architectural distortion at the right side of the sample (H\&E; x100 and x200). C) Trophozoites between villi (H\&E; x200). D) 'Smiley face' like appearance (H\&E; x1000).

shaped morphology, it can be easily overlooked especially in faintly stained slides in cases with fewer trophozoites because of its resemblance to desquamated surface epithelial cells.

E. histolytica is also a 10 to $60 \mu \mathrm{m}$ parasite and the trophozoites can easily be overlooked as they usually reside in the superficial mucosa or in the lumen admixed with cell debris, and can thus easily be mistaken as desquamated epithelial cells. Overlooking the trophozoites can be misleading for the differential diagnosis of the case with chronic inflammatory bowel disease (IBD) as amebiasis can mimic the histopathological changes seen in ulcerative colitis or Crohn's disease, the misdiagnosis of which may result in treatment with steroids that would only exacerbate the infection (6). In this respect, all cases in our series showed chronic active inflammation on superficial evaluation, while half of them also presented with ulcero-erosive lesions, mucosal architectural changes and eosinophilic infiltration that are also common features of IBD. However, none of them showed basal plasmacytosis, which is a cardinal feature of IBD or crypt abscess, granuloma formation, or pseudopyloric or Paneth cell metaplastic changes that are also encountered in IBD (1). One should also keep in mind that amebiasis can also coincide or complicate IBD (10) and can be more prevalent especially in cases of ulcerative colitis compared to the normal population (11). In suspicious cases, stool microscopy or rectal swabs can be very helpful before making the diagnosis or when planning a therapeutic scheme.

A similar histology with chronic lymphoplasmacytic infiltration was also observed in our E. vermicularis cases accompanied by cryptitis, erosion and ulcerations. E. vermicularis usually resides in the caecum, appendix and distal ileum and thus can also mimic Crohn's disease in 

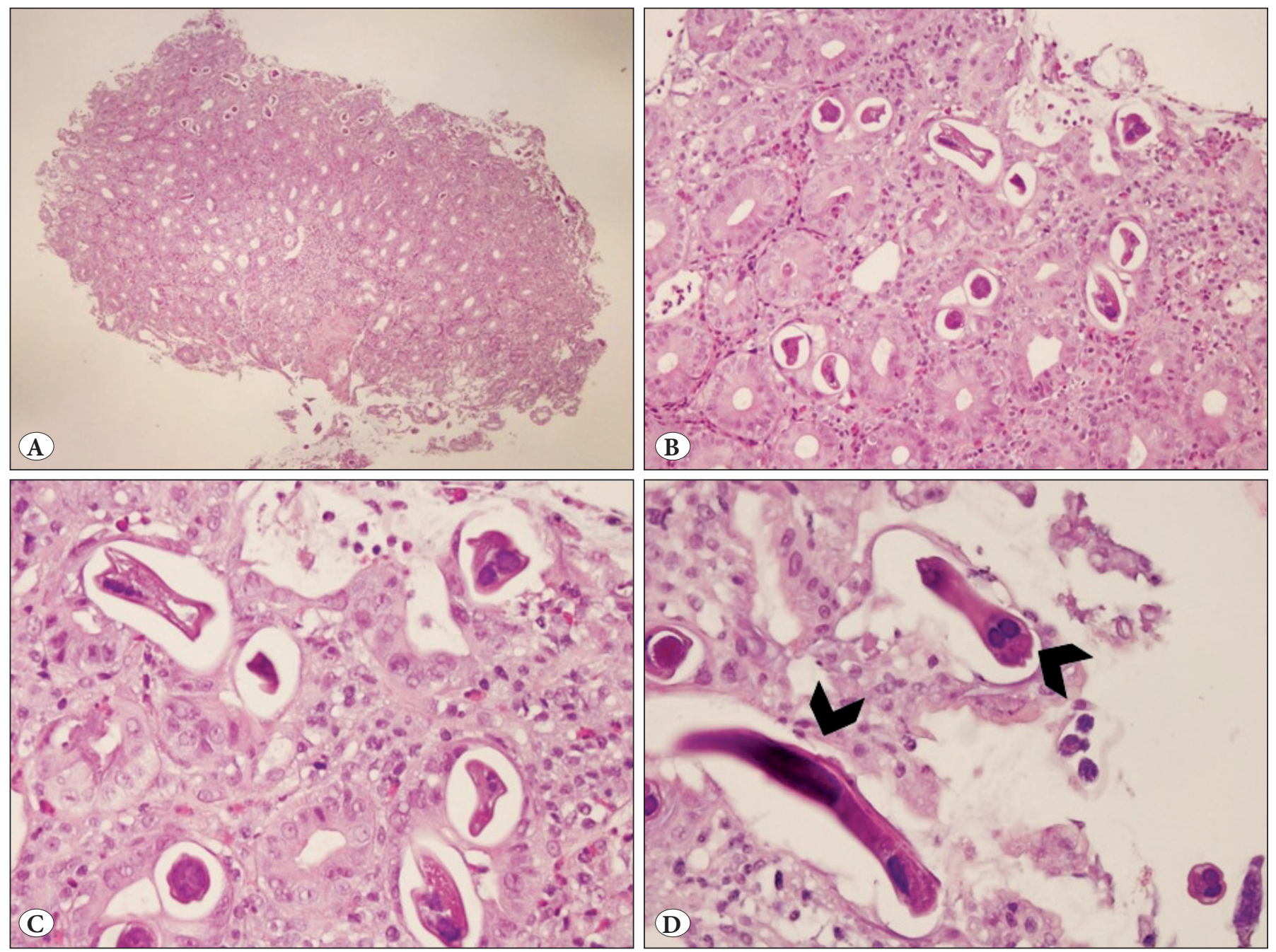

Figure 5: A-D) Strongyloides larvae in gastric biopsy (arrows) (H\&E; x40, x200,x400 and x400).

this context (3). However, no granuloma formation was observed and mucosal architectural changes such as crypt distortion were not prominent. Additional findings were hyalinization and calcification that had probably developed secondary to ulceration, necrosis and perforation present in the index cases. Although the clinical history of enterobiasis, which is commonly referred to as pinworm, is very typical with anal pruritus, histopathological diagnosis can be troublesome when the parasite is very sparse, fragmented or unevenly distributed as it can easily be overlooked in cases with extensive phlegmonous inflammation especially in the appendix. In contrast, there may be very limited inflammation when the worms lie only in the lumen (12), again posing diagnostic difficulties. In cases with limited amounts of parasite or inflammation, sampling errors are commonly encountered. However, when provided with clinical information, it will not be much of a burden for the pathologist to examine the entire specimen to find areas with more easily identifiable parasites. In this respect, biopsy materials are much more restrictive and may be less informative depending on the area where the biopsy is taken.

Hyalinization, necrosis and perforation were also observed in the taeniasis case but probably developed due to ischemia caused by bowel obstruction while the other inflammatory changes were even more subtle than in $G$. intestinalis infection. Interestingly, the gastric mucosa with Strongyloides infection was almost normal other than the presence of the parasite itself. The only mucosal alteration was eosinophilic infiltration with 17 and 21 eosinophils/ HPF in the small bowel with taeniasis and the gastric mucosa with Strongyloides, respectively.

The most flamboyant histology was observed in the fascioliasis case with necrosis, perforation, abscess and granuloma formation, giant cells and Charcot-Leyden 

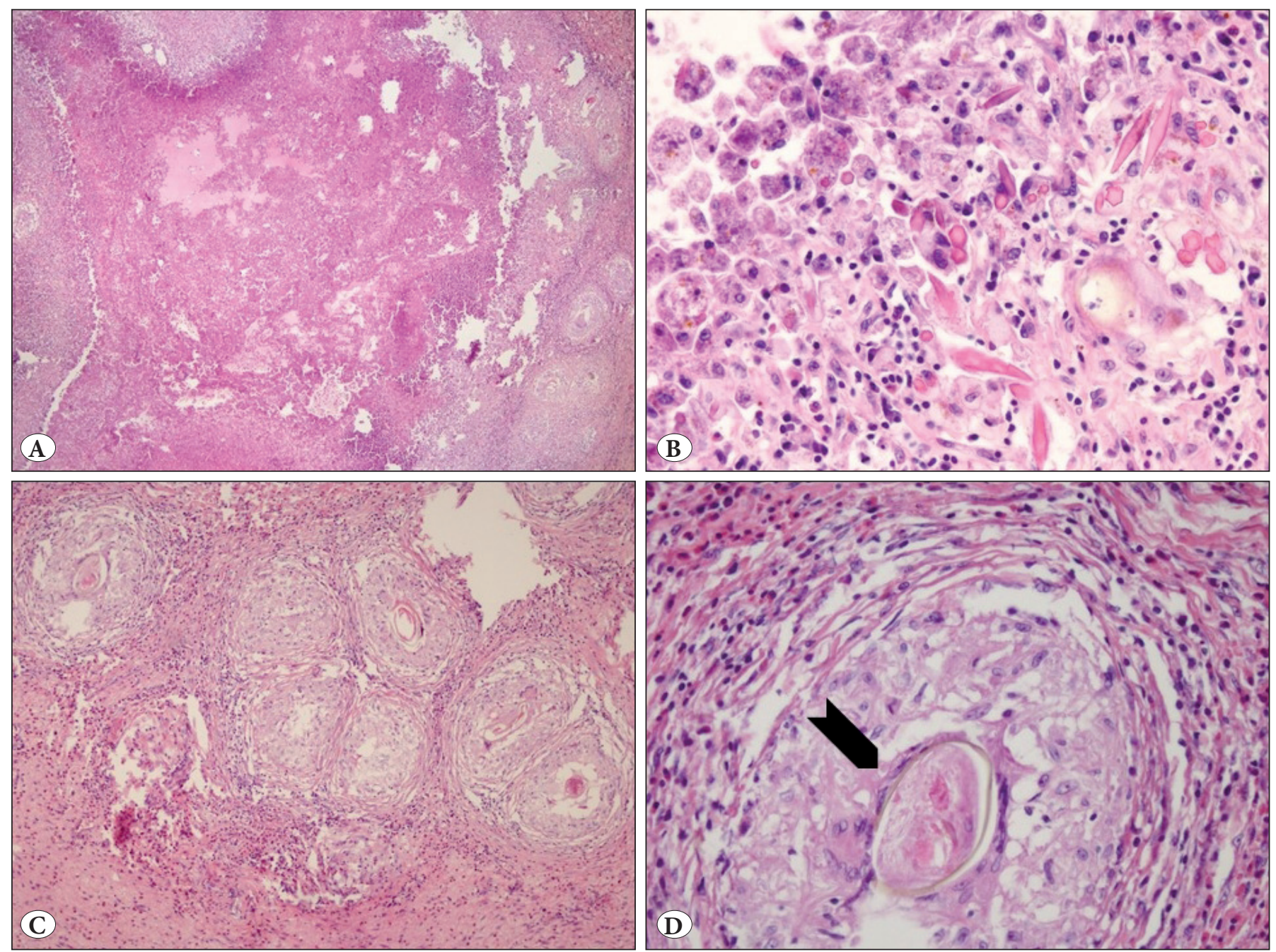

Figure 6: Ascending colon resected due to Fasciola hepatica infection. A) Geographic ulcers caused by adult form of the parasite and granuloma formation (H\&E; x40). B) Charcot-Leyden Crystals and histiocytic infiltration at the edge of the ulcers (H\&E; x200). C) Granulomas containing F. hepatica eggs and giant cells (H\&E; x100). D) F. hepatica egg with a yellowish shell (arrow), at the center of a granuloma (H\&E; $\mathrm{x} 400)$.

crystals. Interestingly, although many of the cases discussed herein had eosinophilic infiltration to some extent, only the fascioliasis case showed prominent Charcot-Leyden crystals.

Overall, the most common accompanying histological findings were nonspecific and included congestion and non-Peyer patch lymphoid aggregates accompanied by chronic active mucosal inflammation in $37.5 \%$ of the cases. Even eosinophilic infiltration appeared to be a nonspecific finding as it did not exceed 10 eosinophils per one HPF but it was more common with giardiasis among all agents. Erosion and ulceration were features of amebiasis, enterobiasis and giardiasis, possibly due to complicating acute appendicitis and gastric mucinous metaplasia in the latter two.
As the study is limited to the observed protozoa infestations in a 10-years period at a tertiary referral hospital and is mostly composed of clinically or histologically complicated cases, some parasites that are more common in some parts of the world could not be covered under the study due to the lack of cases. However, despite the nonspecific nature of the features observed in this study, this is one of the few studies $(4,13)$ dedicated to searching accompanying histology in parasitic infections.

In conclusion, GI parasitosis should no longer considered as an "exotic" disease as it can also be encountered in industrialized countries (14) as well due to various factors such as increased contact with other cultures, increased travelling and immigration or increased fresh food imports from different parts of the world and particularly from 
developing countries (5). Histopathological examination is crucial not only to diagnose but especially to rule out other GI diseases. Our results show that parasites usually induce a nonspecific chronic or active inflammatory reaction with slight villous or cryptic architectural changes, mild eosinophilic infiltrate, granulomas or CharcotLeyden crystals and they can cause bowel obstruction or perforation. Thus, a pathologist must consider a parasitic infection, especially in cases with IBD-mimicking, Coeliac disease-mimicking areas, which do not fulfill the diagnostic criteria.

\section{REFERENCES}

1. Greenson JK, Lamps LW, Montgomery EA, Owens SR, Polydorides AD, Lauwers GY, Purdy JK, Bihlmeyer SK, Srivastava A. Diagnostic Pathology: Gastrointestinal. 1st ed. Greenson JK, editor. Salt Lake City (UT): Amirsys Inc; 2009.

2. Müller N, von Allmen N. Recent insights into the mucosal reactions associated with Giardia intestinalis infections. Int J Parasitol. 2005; 35:1339-47.

3. Johansson J, Ignatova S, Ekstedt M. Pinworm infestation mimicking Crohn's disease. Case Rep Gastrointest Med. 2013;2013:706197.

4. Gupta E, Bhalla P, Khurana N, Singh T. Histopathology for the diagnosis of infectious diseases. Indian J Med Microbiol. 2009;27:100-6.

5. Masucci L, Graffeo R, Bani S, Bugli F, Boccia S, Nicolotti N, Fiori B, Fadda G, Spanu T. Intestinal parasites isolated in a large teaching hospital, Italy, 1 May 2006 to 31 December 2008. Euro Surveill. 2011;16(24). pii: 19891.
6. Kucik CJ, Martin GL, Sortor BV. Common intestinal parasites. Am Fam Physician. 2004;69:1161-8. PMID:15023017.

7. Koot BG, ten Kate FJ, Juffrie M, Rosalina I, Taminiau JJ, Benninga MA. Does Giardia intestinalis cause villous atrophy in children?: A retrospective cohort study of the histological abnormalities in giardiasis. J Pediatr Gastroenterol Nutr. 2009;49:304-8.

8. Oberhuber G, Kastner N, Stolte M. Giardiasis: A histologic analysis of 567 cases. Scand J Gastroenterology. 1997;32:48-51.

9. Onbaşi K, Günşar F, Sin AZ, Ardeniz O, Kokuludağ A, Sebik F. Common variable immunodeficiency (CVID) presenting with malabsorption due to giardiasis. Turk J Gastroenterol. 2005;16: 111-13.

10. Ozdoğan M, Küpelioğlu A. Crohn's colitis perforation due to superimposed invasive amebic colitis: A case report. Turk J Gastroenterol. 2006; 17:130-2.

11. Ustun S, Dagci H, Aksoy U, Guruz Y, Ersoz G. Prevalence of amebiasis in inflammatory bowel disease in Turkey. World J Gastroenterol. 2003; 9:1834-5.

12. Fenoglio-Preiser CM, Noffsinger AE, Stemmermann GN, Lantz PE, Isaacson PG. Gastrointestinal pathology: An atlas and text. 3rd ed. Fenoglio-Preiser CM, editor. Philadelphia: Lippincott Wiliams\&Wilkins; 2007.

13. Mohamed AE, Ghandour ZM, Al-Karawi MA, Yasawy MI, Sammak B. Gastrointestinal parasites presentations and histological diagnosis from endoscopic biopsies and surgical specimens. Saudi Medical Journal. 2000;21:629-34.

14. World Health Organization (WHO). The world health report 2004: Changing history. Geneva: WHO;2004. 